\title{
Efficient Numerical Analysis of a Periodic Structure of Multistate Unit Cells
}

\author{
Ladislau Matekovits, ${ }^{1}$ Karu P. Esselle, ${ }^{2}$ Mirko Bercigli, ${ }^{3}$ and Rodolfo Guidi ${ }^{3}$ \\ ${ }^{1}$ Dipartimento di Elettronica e Telecomunicazioni, Politecnico di Torino, Corso Duca degli Abruzzi, 24, 10129 Torino, Italy \\ ${ }^{2}$ Engineering Department, Faculty of Science, Macquarie University, Sydney, NSW 2109, Australia \\ ${ }^{3}$ Computational Electromagnetic Laboratory, IDS Ingegneria Dei Sistemi SpA, 56121 Pisa, Italy
}

Correspondence should be addressed to Ladislau Matekovits; ladislau.matekovits@polito.it

Received 22 November 2013; Accepted 9 February 2014; Published 20 March 2014

Academic Editor: Gaobiao Xiao

Copyright (C) 2014 Ladislau Matekovits et al. This is an open access article distributed under the Creative Commons Attribution License, which permits unrestricted use, distribution, and reproduction in any medium, provided the original work is properly cited.

Application of the synthetic function expansion (SFX) algorithm to the analysis of active 1- and 2D periodic structures is presented. The single unit cell consisting of a microstrip line loaded by patches positioned below the line is turned into an active structure by inserting a pair of 2 switches to the two ends of each patch; the states of the pair of switches are changed contemporaneously. Variation of the states of the switches modifies the current distribution on the structure. The tunable multistate unit cell is arranged in 24-, 120-, and $9 \times 24$ element configurations and numerically analyzed. The computational complexity required for the characterization of the large number of possible configurations is lightened by the use of the proposed numerical method.

\section{Introduction}

Metamaterials (MMs) have gained impressive interest in the last decade from many researchers working in different fields of science including electromagnetic (EM) field. The spread of such materials in the EM community has received a substantial input from the largely cited paper [1], which proposes the use of periodic structure for the realization of high impedance surfaces. EM behavior of such realizations exploits the additional, dispersive characteristics resulting from the periodic arrangement of identical elements, called unit cells, with respect to the geometry of the single unit cell in the stand-alone, nonperiodic configuration. The term MM usually refers to these periodic structures, because such configurations are artificially realized, since they are not present in the nature. Analyses of mechanical systems making use of such structures were documented [2] from the early 1950s. 1-, 2-, and 3D realizations have been considered and extensively studied. Interesting and important applications have been devised and implemented, for example, power transmission lines loaded with reactances to reduce losses, but their inherent limitations, for example, narrow bandwidth, limits the characteristics and reduces functionalities.
Next generation MMs are expected to fulfill these limitations. There are different research groups around the word working on micro-, nano- (nanopolymers, ferromagnetic nanowires), and atomic (spin, bianisotropic molecules) scale interventions [3] that make use of the inherent periodic structure of composites (carbon nanotubes) or the position of the atoms in the crystalline structure of the materials. Modifications of the existing periodic structures allow realization of innovative materials and are often named as dispersion engineering [4], because the effects of such interventions on the periodic structure mainly reflect on the dispersion characteristics: that is, phase and group velocity of the propagating wave inside the material can be controlled. This happens to optical, mechanical, acoustic, or EM waves.

More ambitious ideas are considering combination of such interventions at different scales, and multiscale solutions start to appear and to evolve towards applications that have been impossible to be realized even in the very recent past [5]. Such solutions exploit the larger maximal degrees of freedom the innovative materials offer allowing achievement of exclusive properties for novel devices and systems. This idea will be discussed in the present paper, where multiscales are at unit cell level instead of the atomic/nano/microlevels. 
Such approach is not common in the scientific literature, and as discussed below, in some cases it requires the use of unit cells exhibiting multistate features.

Many of the state-of-the-art solutions are static; that is, no possibility of change in the dispersion characteristics during the use of such configurations is possible. If active devices are going to be inserted in the unit cell, the characteristics can be dynamically changed, that is, without changing the physical geometry of the device which remains the same, but changing its EM response through biasing the included active part, and reconfigurable devices are obtained. Examples of such solutions are only sporadically present in the literature: recently published research on leaky wave antennas [5], frequency selective surfaces [6], reflectarrays [7], radomes [8], tunable nanoantenna [9], generation of high-order harmonics [10], and so forth demonstrates the wide range of possible applications. Single reconfigurable magnetic unit cell has also been recently presented [11]. However, to the best of the authors' knowledge no example of an active multiscale element as proposed here has been published in the scientific literature.

The main effect of the change of the EM behavior due to the variation of the biasing of the active devices reflects in the change of the input impedance. Periodic structures are characterized by the so-called Bloch impedance, which take into account both the characteristics of the unit cell and of the periodicity. The Bloch impedance presents a huge variation versus frequency especially near the band gaps; hence wideband matching is a challenging issue. When the characteristics of the periodic structure are changed, as proposed here, the variation of the Bloch impedance must be considered for efficient energy matching/transfer from the source to the device. Insertion of active devices introduces additional problems, since as mentioned above the devices must be biased; that is, their state must be externally controllable. One dimensional realization allows easy introduction of the biasing lines, but this issue becomes more challenging for 2- and 3D geometries.

Devices built up with the (active) multiscale unit cells can be employed in application that requires real-time answer to time and space varying external solicitations, for example, tracking a moving target to be followed by a "smart" antenna of which beam can be scanned and so forth.

On the other hand, the numerical investigation of large, in terms of wavelength, structures is a challenging computational EM issue. During time, different numerical methods have been devised to reduce the computational time and memory occupation. One class of methods is based on subdomain decomposition [12-14] where the overall domain is divided is smaller parts, which are numerically characterized by a reduced number of degrees of freedom. This reduction allows a drastic drop in the computational effort when the overall geometry is computed in a second step. Other techniques as fast multipole expansion [15] also target the reduction of the numerical complexity, but following a different way.

With the recent widespread of periodic structures in the microwave community, the problem of efficient numerical characterization is more and more accentuated, since

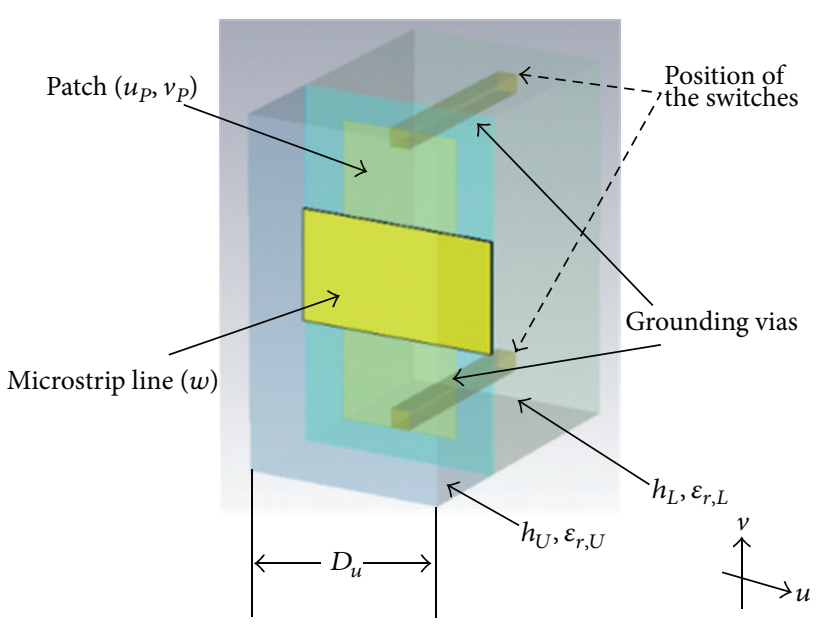

FIGURE 1: Single unit cell.

periodic structures by definition extend to infinity. Practical applications are of limited size, but the structures should contain a large number of unit cells to exhibit the characteristics related to the periodicity, as, for example, the presence of the band gaps. Since the limits of the band gaps correspond to resonances, for an accurate characterization a fine discretization of the geometry is essential. This constrain further increases the number of unknowns, and consequently, the numerical complexity, which motivate the use of dedicated methods that allows efficient handling of the large number of unknowns by reducing the numerical effort.

The complexity of the problem further increases if the analysis regards structures incorporating active devices. Firstly, additional biasing network with respect to the passive case could be present, increasing the metallization to be meshed, that is, the dimension of the system matrix. Secondly the state of the active devices determines the current distribution on the entire structure, implicitly meaning multiple solutions. The computation of all possible current distributions requires remarkable computational resources.

In the present study the multistep synthetic function expansion (SFX) method [14] will be used. It is shortly described in Section 2 together with the geometry. Numerical results are presented and discussed in Section 3 which is followed by a short conclusion.

\section{Descrition of the Unit Cell and Method of Analysis}

2.1. The Unit Cell. In the aforementioned scenario, the present investigation contemplates the numerical analysis of a recently introduced active periodic structure [16]. The considered unit cell shown in Figure 1 consists of a grounded 2-layer configuration, where a microstrip line on the top of the geometry is loaded by a periodic sequence of patches orthogonally positioned with respect to it. The patches positioned below the microstrip line can be selectively connected to the ground plane through two vias. The connection is realized by externally controlled switches. The number of 
possible configurations, determined by the states of the switches, increases exponentially. In order to reduce the computational effort to characterize such a large number of combinations, a subset of the states of the switches, consisting of variable length periodic sequences, have been proposed. The resulting device exhibits the versatility of changing the position and width of the band gaps for different periodic states of the switches [17].

The unit cell is characterized by the longitudinal dimension of $D_{u}=120$ mils. The two dielectric layers have $h_{L}=60$ mils, $h_{U}=13$ mils, respectively. Both layers are characterized by $\varepsilon_{r, U}=\varepsilon_{r, L}=3.3$ and $\tan \delta=0.001$. The unit cell includes the microstrip line of width $w=50$ mils, patches of dimensions $u_{P}=80$ mils and $v_{P}=450$ mils.

2.2. The SFX Method. The technique has been introduced and detailed in [14]. It consists of the separation of the entire structure in smaller parts, called blocks, which are analyzed in unconnected configuration but immersed in between different sources which simulate hypothetical metallizations around. This approach allows numerically defining entire domain basis functions which incorporate effects of presence of virtual metallization, that is, coupling, taking into account possible external sources. The current distributions obtained for different excitations (natural, connection, and coupling), targeting to represent all possible interactions with metallization external to the block, are orthogonalized and named as synthetic functions (SFs). In the following step, a reduced number of SFs-corresponding to the minimum degree of freedom required to the correct representation of the solution-are used as entire domain basis functions to discretize the current distribution on the overall structure. Finally, the solution in the reduced based is expanded to obtain the current distribution in the initial subdomain representation. The scheme drastically reduces the dimension of the method of moments (MoM) matrix, which in turn reflects in the reduction of the solution time and memory resources needed for the analysis [14]. In [18], it has already been demonstrated that the iteration-free SFX technique is well adapted to the analysis of passive periodic structures $[19,20]$. Here it will be applied for different 1- and 2D configurations built up with the unit cell in Figure 1 and used to reduce the computational effort when the incorporated switches are in "on" and "off" states, respectively.

2.3. Generation of the SFs. Since the structure offers the opportunity to selectively control the loads by changing the states of the pair of switches attached to each patch, a complete analysis for all possible combinations, even with a nonperiodic control pattern of the switches, results to be a very time-consuming one. This effort is alleviated here by the use of the SFX method. For the generation of the entire domain basis function, the simplest configuration consists of considering the single element in the stand-alone configuration, and this approach has been considered here. This choice contemplates computation of the SFs only for one block and uses such distributions for all the other blocks. The

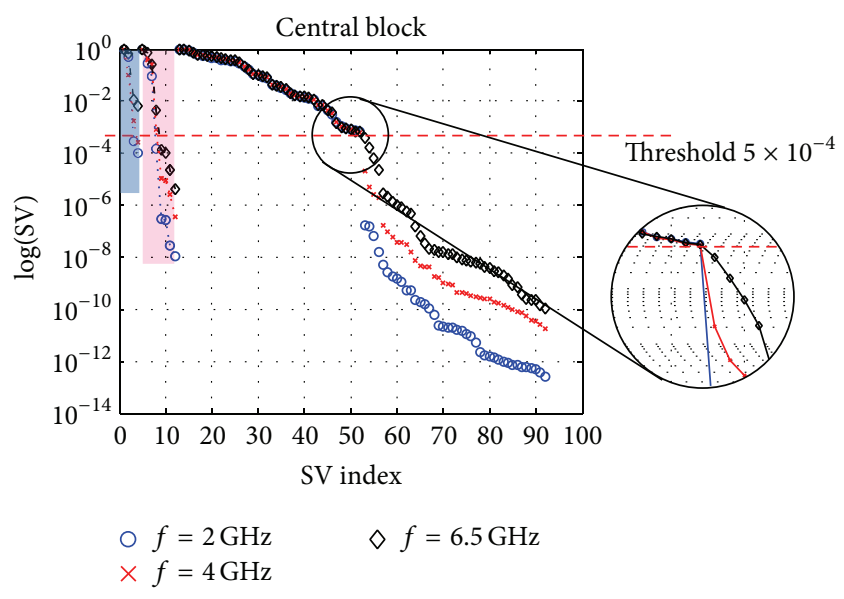

FIGURE 2: Distribution of the singular values for different frequencies for the single central block.

block-Toeplitz structure of the MoM matrix has also been considered when possible during the analysis.

The numerical analysis has been carried out using the commercial software [21]. Each block has been discretized by 218 RWG 4 PWL and 2 attachment basis functions. A total of $2 \times 10=20$ auxiliary sources have been distributed on two rectangular layouts at the two metallization levels. The first and last blocks have been treated separately and differently with respect to the central blocks, since they are fed/loaded externally. At the edge of the internal blocks connection functions have been considered. The first and last blocks have a connection function section where they are united to the rest of the circuit and natural ports at the other end.

Each block has been considered in stand-alone configuration, and the responses to the external excitation have been computed. The obtained current distributions form the basis for the reduced representation. The next step consists of orthogonalizing the basis and retains only the most important terms. The orthogonalisation has been carried out by the use of the singular value decomposition. The evolution of the singular values for a central block for different frequencies is reported in Figure 2. The corresponding singular vectors correspond to the synthetic functions (SFs). Similar behavior can be observed for the different frequencies which allow selecting a quite stable number of SFs. Consequently, it is expected that the analysis at various frequencies will require the same CPU time.

The above described procedure has been applied to the other two types of blocks too. Similar behavior has been observed and will be not reported here. In the following analysis we have considered a threshold level of $5 \times 10^{-4}$. As a result, for each end/central block, besides the $2 / 4$ natural functions, 3 connection and 40 auxiliary SFs have been considered. This guarantees a reduction from the initial 224 basis functions to $45 / 47$ functions per each block. In the final system, this number is amplified by the number of considered blocks.

The presence of active devises are taken into account as follows: the connection points between switches and circuit 


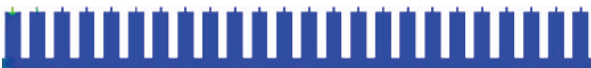

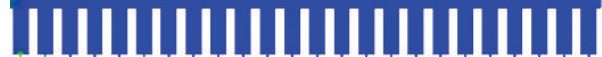

(a)

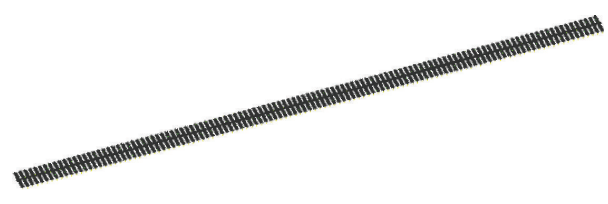

(b)

Figure 3: 1D 24-element array (a) and 120-element array (b).

elements are considered as natural ports and are excited in order to enlarge the rank of the solution space by these additional basis functions. Consequently, the current distributions generated considering these ports allow modelling the change in the solution for the different states of the switches (which are unknown when SFs are generated).

\section{Analysis, Results and Discussions}

3.1. 1D Cases. Different configurations have been considered. First a short $N_{C 1}=24$ element configuration has been analyzed, which has been followed by a longer $N_{C 2}=$ 120 element arrangement. Both configurations are shown in Figure 3.

The comparison in terms of scattering parameters for different switch configurations (i) all "on," (ii) all "off," and (iii) sequence of "on"-"off" for the 24-element array between standard MoM solution and SFX approach was very good in the considered $3-8 \mathrm{GHz}$ frequency range, which covers the first pass-, first stop, and second passbands and fits the experimental results in [17]. The corresponding surface current distributions at $2.9 \mathrm{GHz}$ (in the first passband) and $f=3.9 \mathrm{GHz}$ (in the stop band) are reported in Figure 4. This second frequency is very close to the beginning of the stop band (but already within the stop band) for both configurations that exhibit this behavior.

For the analysis of the active solutions, analogously to the analysis in [16], the state of the switches are modeled as connection between the vias and ground plane for the "on" state of the switches and as a missing metallization for the "off" state. The insertion of such conditions in the solution is done at the scattering matrix level; that is, the S-matrix of the overall system of dimension $2 \times N_{C}+2=50$ and 242, respectively, is reduced to the $2 \times 2$ matrix corresponding to the 2-port structure defined by the input-output ports at the two ends of the microstrip line. The active solution in terms of current is obtained by means of a proper linear combination of the overall currents solution space. Since this approach does not require solving any system, it is very fast.

The difference in the current distribution corresponding to altered states of the switches is reported in Figure 5. Here a longer, 120-element array has been analyzed at a frequency of $6.5 \mathrm{GHz}$, while the all "on" case correspond to a situation where the mode is below the light line, that

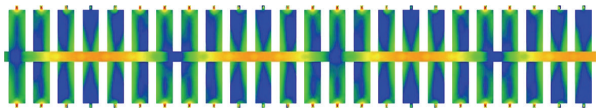

(a)

\section{曲冊冊冊曲冊州}

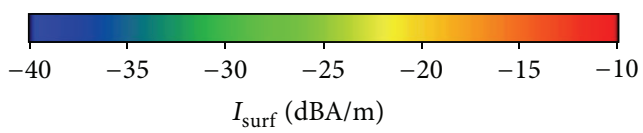

(b)

FIGURE 4: 24-element array: surface current distribution obtained by the SFX method at $f=2.6 \mathrm{GHz}(\mathrm{a}), f=3.9 \mathrm{GHz}(\mathrm{b})$.

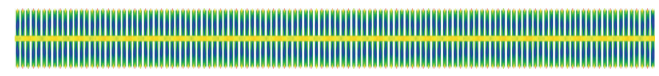

(a)
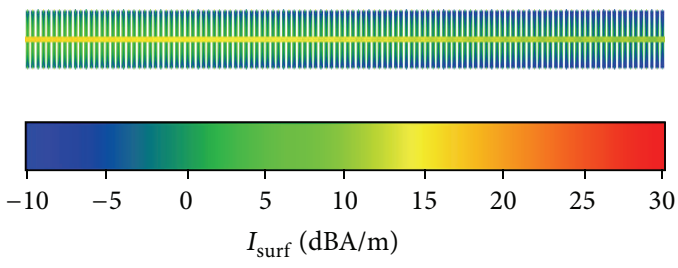

(b)

FIGURE 5: 120-element array: surface current distribution obtained by SFX method at $f=6.5 \mathrm{GHz}$. All "on" (a) and all "off" configuration (b).

is, no radiation exists, for the all "off" case the structure radiates. The comparison of the current distributions for a longer configuration makes in evidence the leakage when all switches are in "off" state. No leakage is present for the all "on" state case. This phenomenon is accurately taken into account by the proposed numerical scheme.

3.2. $2 \mathrm{D}$ Case. For the $2 \mathrm{D}$ case the short 24-element structure has been considered and repeated for a total of 9 times in the orthogonal to the microstrip direction. The surface current at $6.5 \mathrm{GHz}$ for all switches in "on" state is reported in Figure 6. In the analysis, only the central microstrip was fed. All other ports were loaded by $50 \Omega$. The presence of the second periodicity still allows propagation at this frequency. A strong coupling between the different parallel structures can be observed in Figure 6(a). A closer view in the central plot makes it clear that the coupling is due to the surface waves generated by the fed structure.

3.3. The Computational Effort. Computations have been carried out on computers equipped with x86 Family 6 model 15 Genuine Intel, CPU (2659 MHZ), 4 GB memory, Windows(R) XP 32bit operating system. For the 120-element 


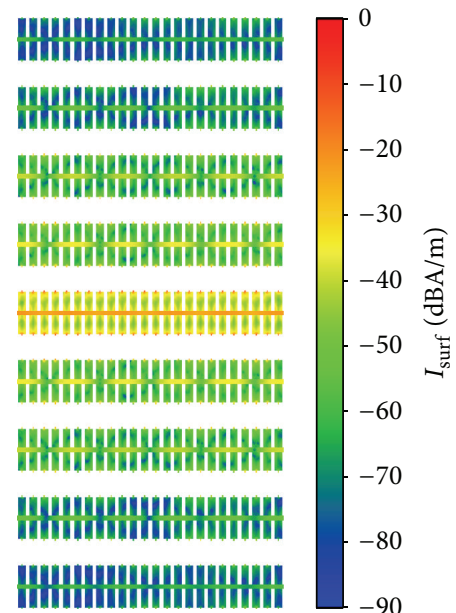

(a)

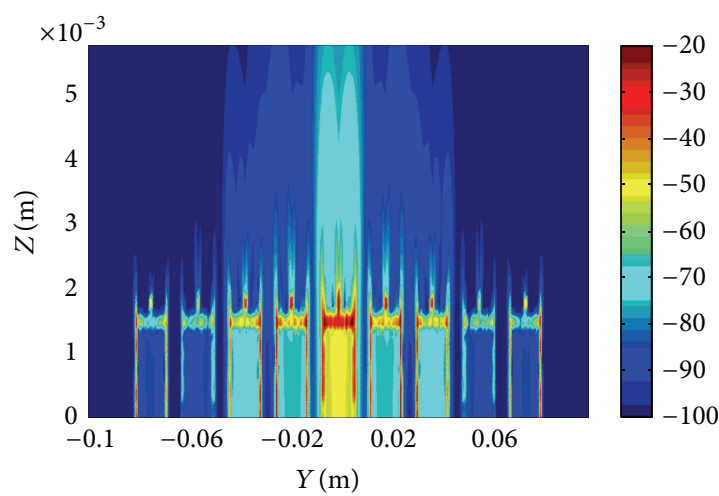

(b)

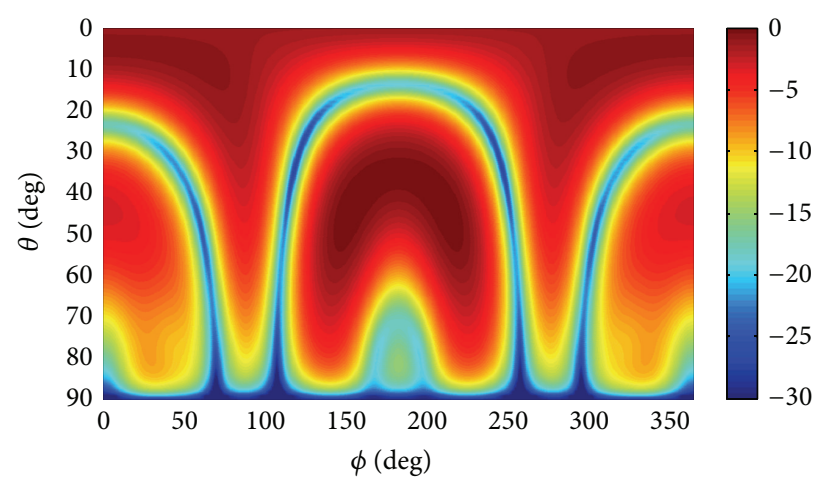

(c)

Figure 6: $9 \times 24$-element 2D array, all "on" switch configuration, results at $f=6.5 \mathrm{GHz}$ : surface current distribution (a) and electric field distribution in a plane orthogonal to the microstrip line near field (b) and far field (c).

TABLE 1: Comparison between MoM and SFX numerical efforts (for 1 frequency point).

\begin{tabular}{|c|c|c|c|c|c|c|}
\hline & \multirow{2}{*}{\multicolumn{2}{|c|}{$\begin{array}{l}\text { Single precision } \\
\text { 24-element array }\end{array}$}} & \multirow{2}{*}{\multicolumn{2}{|c|}{$\begin{array}{c}\text { Double precision } \\
9 \times 24 \text {-element array }\end{array}$}} & \multirow{2}{*}{\multicolumn{2}{|c|}{$\begin{array}{l}\text { Double precision } \\
120 \text {-element array }\end{array}$}} \\
\hline & & & & & & \\
\hline & MoM & SFX & MoM & SFX & MoM & SFX \\
\hline $\begin{array}{l}\text { Unknowns } \\
\text { CPU }\end{array}$ & 5391 & $\begin{array}{c}1220 \\
12 \mathrm{~min}\end{array}$ & 48.519 & $\begin{array}{c}11880 \\
3 \mathrm{~h} 5 \mathrm{~min}\end{array}$ & $\begin{array}{c}26.895 \\
9 \text { h } 11 \mathrm{~min}\end{array}$ & $\begin{array}{c}6116 \\
33 \mathrm{~min}\end{array}$ \\
\hline RAM & & 60 MByte & & 1 GByte & 6 GByte & 0.6 GByte \\
\hline
\end{tabular}

array a Xeon X5667 quad-core CPU (3070 MHz), $96 \mathrm{~GB}$ memory, Windows Server 2003 Enterprise x64 edition operating system configuration has been used.

A comparison in terms of CPU time and memory requirement for the different configurations discussed above is reported in Table 1 . The data refer to the computation of the surface current distribution for a single frequency point.

As expected, the reduction of both CPU time and memory allocation has been achieved. Moreover, no frequency dependence of the monitored quantities has been observed for the considered cases.

In the case of an active circuit, the structure of the blocks varies versus the state of the active devices which in turn modifies the current distribution too. With standard techniques, the determination of the different current distributions requires additional computational effort, as, for example, solution of multiple right-hand side systems. In these cases, even if only the back substitution is considered, for large matrixes this results to be very time consuming. Contrarily, in the case of the SFX approach, which intrinsically considers a basis able to represent any possible current distributions within the block, the required numerical effort is fundamentally the same.

\section{Conclusions}

Application of the SFX method to 1- and 2D active periodic structures has been presented. The reconfigurable devices 
have been divided into identical blocks and analyzed for different states of the switches. Drastically reduction in the computational resources without accuracy loss with respect to standard MoM solution has been demonstrated. The method allows fast analysis of the circuit behavior for different switch patterns, which can be efficiently exploited in combination with optimization tools.

\section{Conflict of Interests}

The authors declare that there is no conflict of interests regarding the publication of this paper.

\section{Acknowledgment}

The research has been supported by an Australian Research Council funded Discovery Project (DP130102009).

\section{References}

[1] D. Sievenpiper, L. Zhang, R. F. Jimenez Broas, N. G. Alexöpolous, and E. Yablonovitch, "High-impedance electromagnetic surfaces with a forbidden frequency band," IEEE Transactions on Microwave Theory and Techniques, vol. 47, no. 11, pp. 2059-2074, 1999.

[2] L. Brillouin, Wave Propagation in Periodic Structures, Dover, New York, NY, USA, 1953.

[3] J. Henzie, M. H. Lee, and T. W. Odom, "Multiscale patterning of plasmonic metamaterials," Nature Nanotechnology, vol. 2, no. 9, pp. 549-554, 2007.

[4] C. Caloz, "Metamaterial dispersion engineering concepts and applications," Proceedings of the IEEE, vol. 99, no. 10, pp. 17111719, 2011.

[5] R. O. Ouedraogo, E. J. Rothwell, and B. J. Greetis, "A reconfigurable microstrip leaky-wave antenna with a broadly steerable beam," IEEE Transactions on Antennas and Propagation, vol. 59, no. 8, pp. 3080-3083, 2011.

[6] F. Bayatpur and K. Sarabandi, "Design and analysis of a tunable miniaturized-element frequency-selective surface without bias network," IEEE Transactions on Antennas and Propagation, vol. 58, no. 4, pp. 1214-1219, 2010.

[7] T. H. Hand and S. A. Cummer, "Reconfigurable reflectarray using addressable metamaterials," IEEE Antennas and Wireless Propagation Letters, vol. 9, pp. 70-74, 2010.

[8] T. Jiang, Z. Wang, D. Li et al., "Low-DC voltage-controlled steering-antenna radome utilizing tunable active metamaterial," IEEE Transactions on Microwave Theory and Techniques, vol. 60, no. 1, pp. 170-178, 2012.

[9] Z. Gong, T.-W. Lee, and L. Que, "Design and modeling of a MEMS-based tunable optical nanoantenna," in Proceedings of the IEEE 14th International Mixed-Signals, Sensors, and Systems Test Workshop, (IMS3TW'08), June 2008.

[10] Z. Wang, Y. Luo, L. Peng et al., "Second-harmonic generation and spectrum modulation by an active nonlinear metamaterial," Applied Physics Letters, vol. 94, no. 13, Article ID 134102, 2009.

[11] L. Jelinek and J. MacHac, "An FET-based unit cell for an active magnetic metamaterial," IEEE Antennas and Wireless Propagation Letters, vol. 10, pp. 927-930, 2011.

[12] V. V. S. Prakash and R. Mittra, "Characteristic basis function method: a new technique for efficient solution of method of moments matrix equations," Microwave and Optical Technology Letters, vol. 36, no. 2, pp. 95-100, 2003.

[13] E. Suter and J. R. Mosig, "A subdomain multilevel approach for the efficient MoM analysis of large planar antennas," Microwave and Optical Technology Letters, vol. 26, pp. 270-277, 2000.

[14] L. Matekovits, V. A. Laza, and G. Vecchi, "Analysis of large complex structures with the synthetic-functions approach," IEEE Transactions on Antennas and Propagation, vol. 55, no. 9, pp. 2509-2521, 2007.

[15] J. Song, "Multilevel fast multipole algorithm for electromagnetic scattering by large complex objects," IEEE Transactions on Antennas and Propagation, vol. 45, no. 10, pp. 1488-1493, 1997.

[16] L. Matekovits, M. Heimlich, and K. Esselle, "Tunable periodic microstrip structure on GaAs wafer," Progress in Electromagnetics Research, vol. 97, pp. 1-10, 2009.

[17] D. N. P. Thalakotuna, K. P. Esselle, L. Matekovits, M. Heimlich, and S. G. Hay, "Changing the electromagnetic bandgap and stopbands in a multistate periodic circuit," Microwave and Optical Technology Letters, vol. 55, no. 81871, pp. 1871-1874, 2013.

[18] L. Matekovits, G. Vecchi, M. Bercigli, and M. Bandinelli, "Efficient numerical analysis of large planar high impedance surface by the Synthetic Function eXpansion technique," Microwave and Optical Technology Letters, vol. 51, no. 11, pp. 2763-2769, 2009.

[19] L. Matekovits, G. C. V. Colomé, and M. Orefice, "Controlling the bandlimits of TE-surface wave propagation along a modulated microstrip-line-based high impedance surface," IEEE Transactions on Antennas and Propagation, vol. 56, no. 8, pp. 2555-2562, 2008.

[20] L. Matekovits, G. Vecchi, M. Bercigli, and M. Bandinelli, "Synthetic-functions analysis of large aperture-coupled antennas," IEEE Transactions on Antennas and Propagation, vol. 57, no. 7, pp. 1936-1943, 2009.

[21] ADF-EMS website, https://www.idscorporation.com/images/ Downloads/Space/SPACE_ADF-EMS.pdf. 

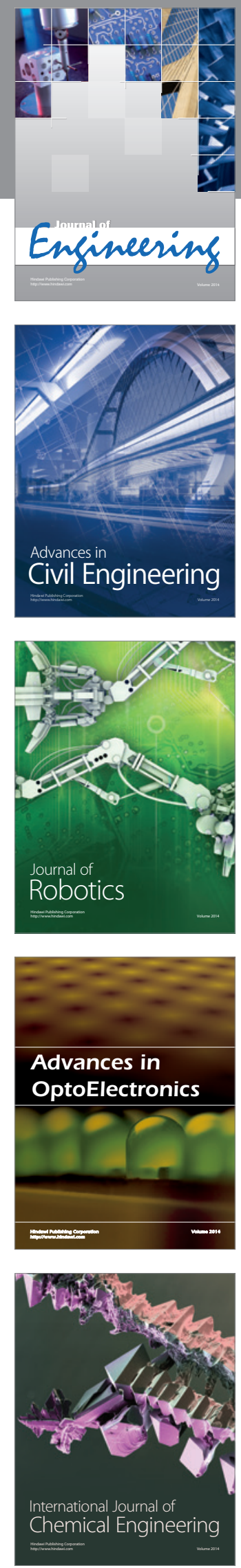

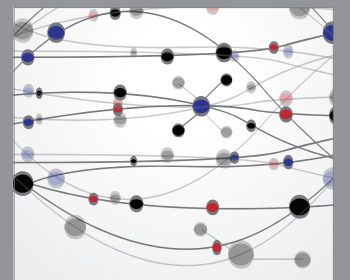

The Scientific World Journal
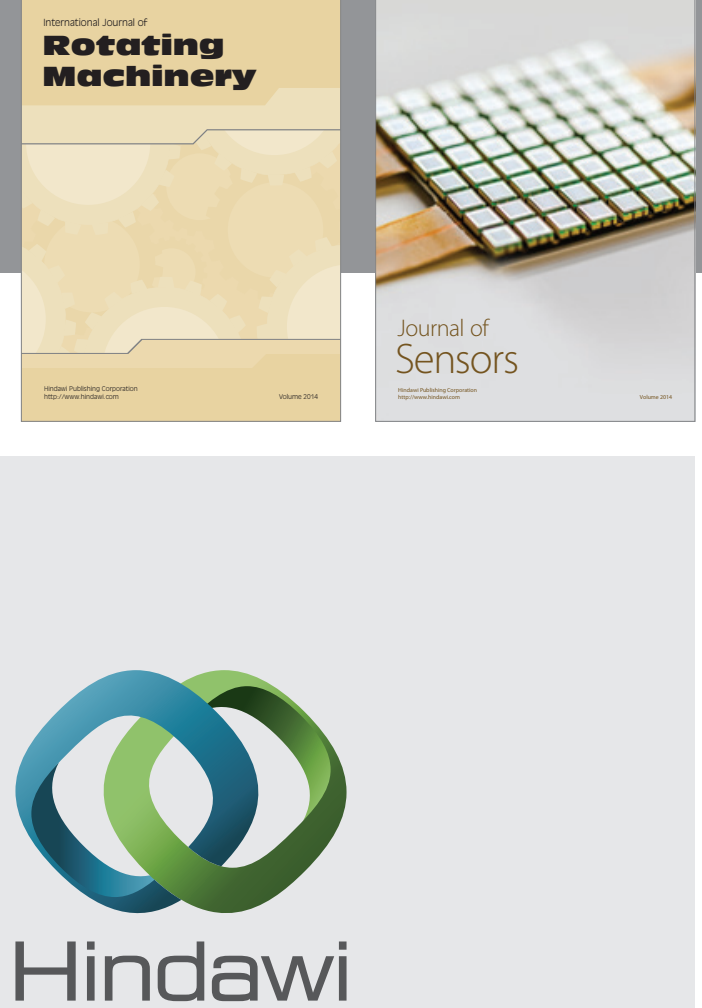

Submit your manuscripts at http://www.hindawi.com
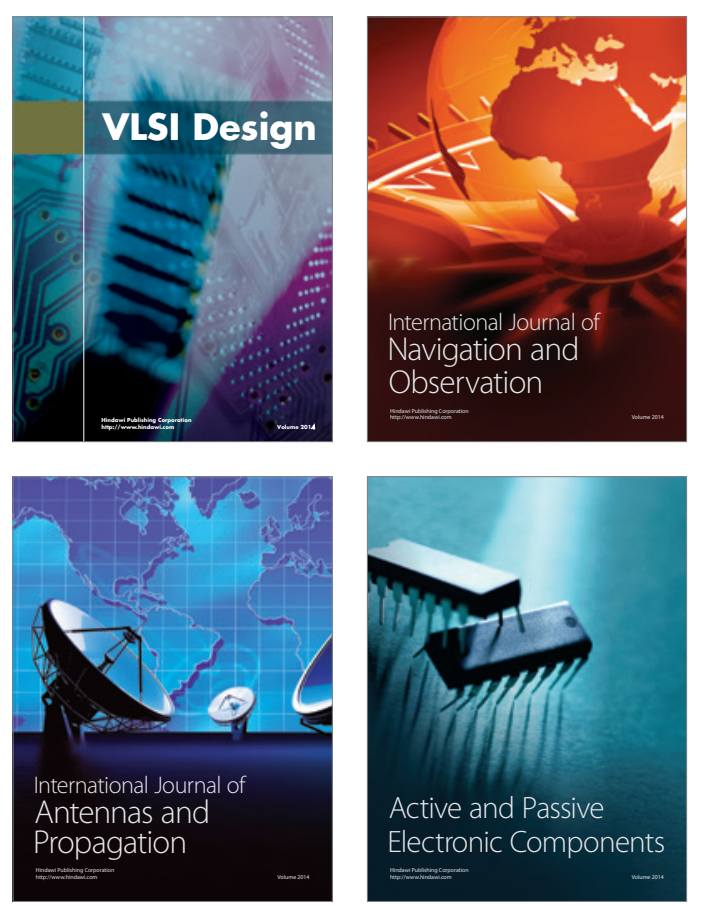
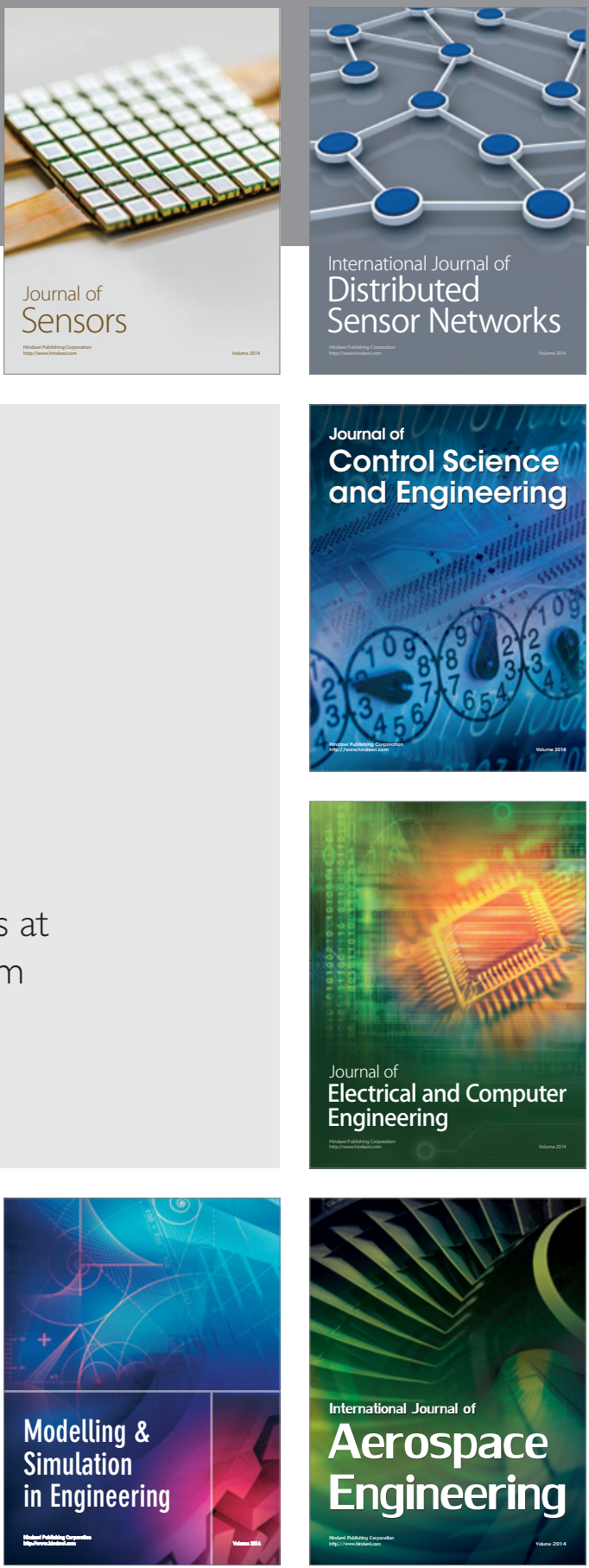

Journal of

Control Science

and Engineering
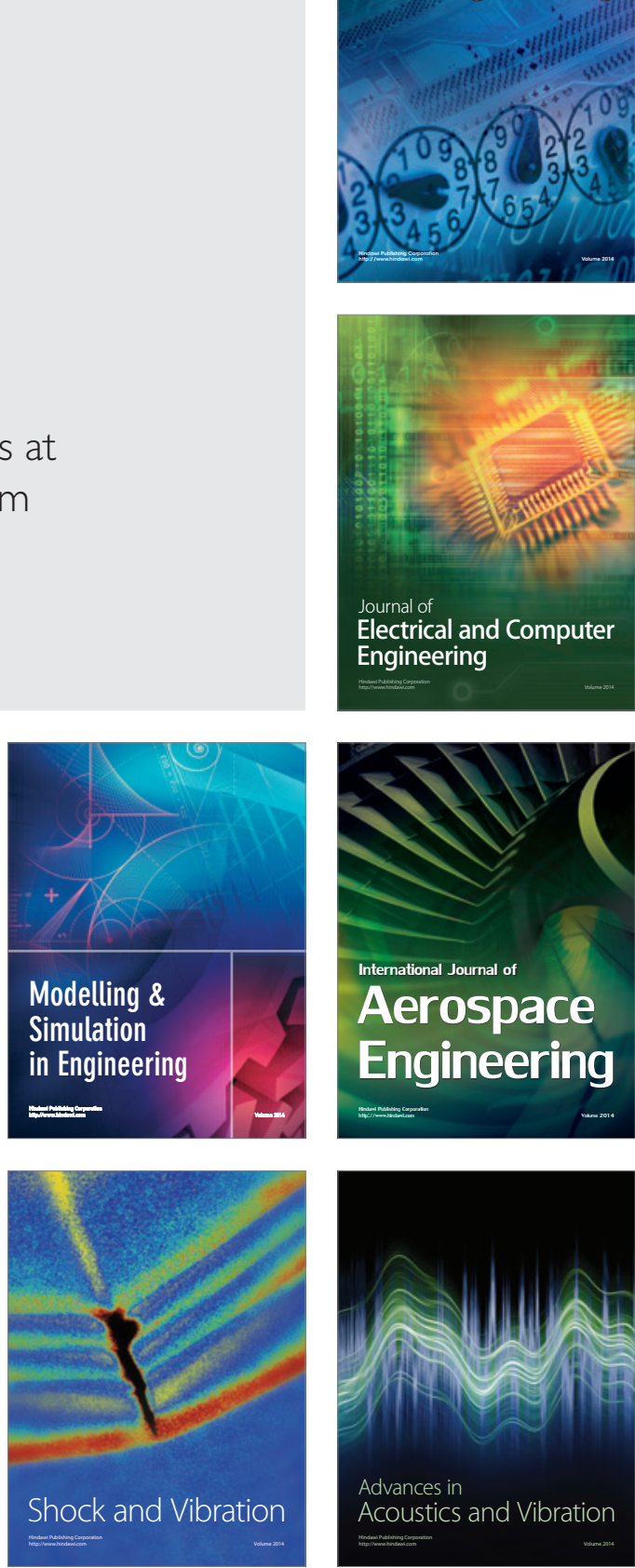